\title{
Synthesis of an Imidazolidinone Organocatalyst and Its Application in a Diels-Alder Cycloaddition: A Multistep Experiment for the Organic Teaching Laboratory
}

\author{
John J. Murphy, Ross B. Driver, Ria Walsh, and John C. Stephens* \\ Department of Chemistry, Maynooth University, Maynooth, Co. Kildare, Ireland
}

Supporting Information

\begin{abstract}
The development of novel, high-yielding, and selective methodologies for the asymmetric synthesis of stereocenters is at the forefront of modern synthetic chemistry research. Organocatalysis can now be viewed as a viable alternative to the use of the sometimes toxic transition-metal

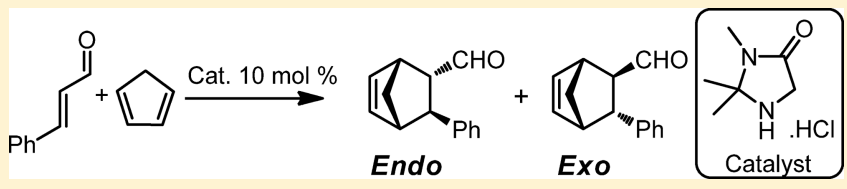
catalysts. In this experiment, the simple synthesis of an achiral imidazolidinone organocatalyst is described and its performance is compared to that of a related commercially available chiral catalyst in a Diels-Alder cycloaddition. The organocatalyst operated via an iminium ion intermediate and the product of the Diels-Alder cycloaddition was purified by silica plug filtration (no flash column chromatography required). The diastereoselectivity was readily measured with ${ }^{1} \mathrm{H}$ NMR spectroscopy and optionally, the enantioselectivity of the chiral catalyst can be assessed with chiral GC. The experiment can be performed over three laboratory sessions and is suitable for a third- or fourth-year undergraduate laboratory curriculum. The experiment demonstrated not only the synthesis of an organocatalyst but also the effect and importance of catalyst design on the stereochemical outcome of a Diels-Alder cycloaddition.

KEYWORDS: Upper-Division Undergraduate, Organic Chemistry, Analytical Chemistry, Laboratory Instruction, Hands-On Learning/Manipulatives, Asymmetric Synthesis, Catalysis, Chromatography, Spectroscopy, Synthesis
\end{abstract}

$\mathrm{T}$ he term organocatalysis has become synonymous with metal-free asymmetric catalysis ever since MacMillan coined the phrase in his landmark publication in $2000 .^{1}$ The importance of the use of organic molecules as catalysts, for both symmetric and asymmetric transformations, is highlighted by the large number of papers, review articles, and books published on the subject since 2000 . $^{2,3}$ Early success in this field led to an unprecedented level of research over the past decade, resulting in the development of a host of reactions and an abundance of catalysts. $^{2,3}$

The importance and presence of organocatalysis in the undergraduate curriculum has increased over the past few years. $^{4-14}$ This is due to the prominence of organocatalysis in the literature and its increasing use in industrial processes. The imidazolidinone ring, as a scaffold for aminocatalysis, has garnered much attention due to its rather facile synthesis from convenient chiral starting materials and its ability to form reactive intermediates through manipulation of the HOMO, LUMO, and SOMO energy levels after condensation with a carbonyl substrate. ${ }^{15}$ The laboratory practical described herein involves the synthesis of an imidazolidinone organocatalyst and its application in a Diels-Alder cycloaddition. ${ }^{16}$ The practical was taken by third- and fourth-year undergraduate chemistry students (BSc Science, MH201) at Maynooth University and as such has been run twice (11 third-year students and 8 fourthyear students; all students worked individually). During the practical, the students synthesized the achiral imidazolidinone 1 (Figure 1), 2,2,3-trimethylimidazolidin-4-one hydrochloride, in

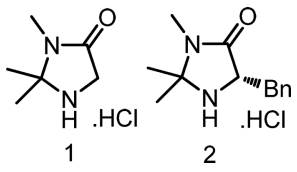

Figure 1. Achiral imidazolidinone $\mathbf{1}$ and commercial chiral imidazolidinone (MacMillan's catalyst) 2.

two steps (Scheme 1) and employed the achiral imidazolidinone $\mathbf{1}$ in the iminium ion promoted Diels-Alder cycloaddition between cinnamaldehyde and cyclopentadiene (Scheme 2).

A comparison of the structure and performance of achiral imidazolidinone $\mathbf{1}$ to those of the commercial chiral imidazolidinone 2 (Figure 1), (5S)-2,2,3-trimethyl-5-benzyl-4imidazolidinone hydrochloride, allows the student to gain an understanding of the importance and role of organocatalyst structure in stereochemical outcome. In addition, comparison with an uncatalyzed reaction helped students understand the role a catalyst plays in providing an alternative reaction pathway with a lower activation energy barrier. The Diels-Alder reaction background rate (without catalyst) was confirmed to be negligible in an experiment performed by a laboratory assistant. The instructor can tailor the practical to suit his/her

Received: October 7, 2015

Revised: June 5, 2016

Published: June 27, 2016 
Scheme 1. Synthesis of Achiral Imidazolidinone 1

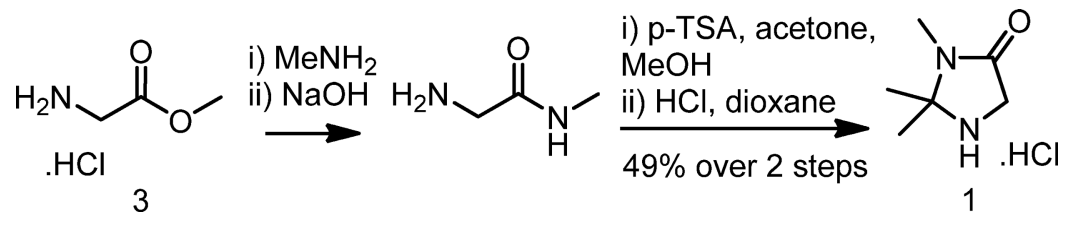

Scheme 2. Iminium Ion Catalyzed $[4+2]$ Cycloaddition

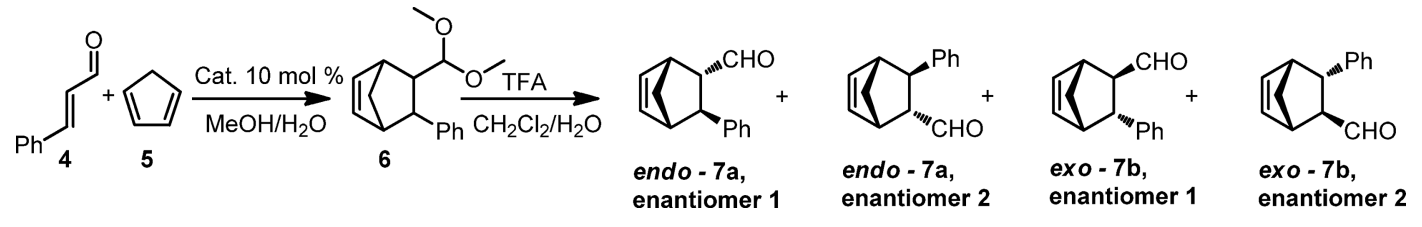

needs, allowing him/her to highlight themes from the experiment that are relevant to their pedagogical goals and course content. The laboratory exercise allows the student to learn about topics including amide synthesis, aminal synthesis, acetal formation, organocatalysis, enantioselectivity, NMR spectroscopy, chiral GC, TLC, Diels-Alder cycloadditions, and the primary literature. The pedagogical goals at Maynooth University were to allow students to gain skills in multistep synthesis, which involved the generation of a target molecule (catalyst), knowing it is to be used in a subsequent application (Diels-Alder reaction). More specifically, the students gain an understanding of the importance of good laboratory practice and the consequences of careless experimental work for later steps as well as the development of a detailed and practical knowledge of iminium mediated organocatalysis and DielsAlder cycloadditions. Several key learning objectives/outcomes were identified for the students that took this practical at Maynooth University. Each student, upon completion of the practical and assessment, was able to:

(1) Demonstrate an understanding of the term organocatalysis,

(2) Demonstrate appropriate use of stereochemical nomenclature,

(3) Describe and illustrate the mechanism for iminium ion formation,

(4) Describe and illustrate the mechanism for an organocatalyzed Diels-Alder cycloaddition,

(5) Demonstrate an ability to structurally characterize a molecule using NMR data,

(6) Demonstrate an understanding of NMR spectroscopy in the determination of diastereoselectivity, and

(7) Demonstrate an understanding of chiral GC in the determination of enantioselectivity.

The success of the students in achieving these learning goals was assessed using a written report and an oral examination. The written report was structured to include a title, date, introduction, results, discussion, experimental section, conclusion, and references. The oral examination was particularly effective, as it motivated students and allowed the examiner to accurately assess student knowledge. Typical oral examination questions are given in the Supporting Information.

Both the organocatalyst synthesis and the Diels-Alder cycloaddition were carried out in a simple fashion without the need for specialized glassware or chromatography purification. Both processes were monitored by TLC with the diastereomeric ratio $(\mathrm{dr})$ determined using ${ }^{1} \mathrm{H}$ NMR spectros- copy and the enantiomeric ratio (er) determined using chiral GC. The dr can also be determined using achiral GC, and an example achiral GC chromatogram (showing separation of the two diastereomers) along with separation conditions can be found in the Supporting Information.

\section{EXPERIMENTAL OVERVIEW}

The two parts of the laboratory practical were completed in 2.5 days (Day one: $3.5 \mathrm{~h}$ session; Day two: $3.5 \mathrm{~h}$ session; Day three: $4 \mathrm{~h}$ session). This, along with the scientific content, makes it an ideal exercise for third- and fourth-year undergraduates. Detailed experimental procedures can be found in the Supporting Information.

\section{Catalyst Synthesis}

The organocatalyst $\mathbf{1}$ was synthesized from the commercially available glycine methyl ester hydrochloride 3 in two steps. The first step consisted of aminolysis of glycine methyl ester hydrochloride over $1.5 \mathrm{~h}$ using a concentrated solution of aqueous methylamine. ${ }^{17}$ Neutralization of the resultant hydrochoride salt of $N$-methylglycinamide, using aqueous sodium hydroxide, allowed for the simple trituration of the free amine product using ethyl acetate. In the second step, an aminalization, the amide was refluxed overnight with acetone and a catalytic amount of $p$-toluenesulfonic acid to give the final imidazolidinone. The imidazolidinone was precipitated using a solution of hydrochloric acid, recrystallized from ethanol in yields of up to $49 \%$ over the two steps (Scheme 1), and characterized using ${ }^{1} \mathrm{H}$ NMR spectroscopy. Students were expected to obtain a catalyst yield of $\sim 40 \%$ from a wellexecuted experiment. The catalyst is a stable crystalline solid at room temperature and can be stored for extended periods of time in a dry atmosphere.

\section{Diels-Alder Reaction}

The Diels-Alder cycloaddition was performed overnight, employing commercial cinnamaldehyde as the pro-dienophile and cracked cyclopentadiene ${ }^{18}$ as the diene. Freshly cracked cyclopentadiene was prepared in advance by a laboratory assistant. The organocatalyst was employed at $10 \mathrm{~mol} \%$ loading in an aqueous methanolic solvent system. Upon consumption of the starting material, as realized by TLC, the reaction mixture was treated with trifluoroacetic acid, stirred for $2 \mathrm{~h}$, neutralized, and extracted into diethyl ether. The trifluoroacetic acid hydrolyzed the acetal of adduct 6 , liberating the final aldehyde. The ethereal extracts (diethyl ether solution of the crude product) were then passed through a pad of silica to give the endo/exo adducts of 7 in high yield (Scheme 2) 
(typical student yield was $80 \%$, yield range was $40-100 \%$ ). It should be emphasized to students that care must be taken to ensure complete removal of residual solvent. Some yields were reported by students as $>100 \%$ due to residual solvent and poor drying. The diastereoselectivity was measured by ${ }^{1} \mathrm{H}$ NMR spectroscopy through comparison of the integrated aldehyde peaks of both the endo and exo products. Separation of the aldehyde endo and exo peaks is sufficient for $\mathrm{dr}$ determination using lower field NMR spectrometers $(60-90 \mathrm{MHz})$. Alternatively, the $\mathrm{dr}$ could be determined by comparison of the endo and exo peaks in the GC chromatogram. The er was determined using chiral-GC.

\section{HAZARDS}

Students must wear safety glasses, lab coats, and gloves. The experiment must be fully risk-assessed by all participants. Methylamine must not be handled outside of a well-ventilated fume hood owing to its pungent odor and flammability. Sodium hydroxide and hydrochloric acid are corrosive. Cinnamaldehyde and cyclopentadiene are irritants. Ethyl acetate, acetone, petroleum ether, and diethyl ether are flammable and can cause serious eye irritation and dizziness upon inhalation. Methanol and ethanol are highly flammable liquids and are toxic if swallowed or inhaled. $p$-Toluenesulfonic acid can cause skin, eye, and respiratory irritation. Hydrochloric acid in 1,4dioxane is highly flammable and causes skin, eye, and respiratory irritation. Trifluoroacetic acid causes severe skin burns and eye damage and is harmful if inhaled. Dichloromethane causes skin and serious eye irritation and may cause respiratory irritation and damage to organs. Chloroform and chloroform-d are harmful if swallowed, cause skin and serious eye irritation, are toxic if inhaled, and cause damage to organs. Neither the product imidazolidinone catalyst 1 nor the adduct 7 has a CAS number or MSDS but should be treated as toxic as a precaution. For a detailed list, see the Supporting Information.

\section{DISCUSSION AND CONCLUSIONS}

The practical exercise allowed students to both synthesize an organocatalyst and to test its effectiveness in a synthetic transformation. Core organic chemistry concepts are covered, including carbonyl chemistry, acetal formation, amines, amino acids, aminals, iminium ions, $\alpha$-carbon reactivity, cycloadditions, stereochemistry, enantioselectivity, and diastereoselectivity. Important analytical techniques such as NMR spectroscopy, IR spectroscopy, TLC, and GC can be focused on. The amount of emphasis placed on each concept and analytical technique is at the instructor's discretion and allows tailoring of the practical to the needs of the student cohort.

The imidazolidinone catalyst $\mathbf{1}$ has not been reported previously to the best of our knowledge, but reactions employing related catalysts, such as MacMillan's imidazolidione 2, have been reported extensively. ${ }^{19}$ The first part of the experiment demonstrated the synthesis of this important class of organocatalyst and employed both an amide bond formation (step 1) and a ring-closing aminalization reaction (step 2). Sufficient quantities of catalyst were produced to allow both analysis and subsequent reaction. The synthesis of the catalyst was monitored by TLC. IR spectroscopy could also have been used to monitor reaction progress, as the carbonyl IR stretch shifts from $1747 \mathrm{~cm}^{-1}$ for the staring glycine ester to $1724 \mathrm{~cm}^{-1}$ for the catalyst (see Supporting Information for spectra and
TLC $R_{\mathrm{f}}$ values). An example student ${ }^{1} \mathrm{H}$ NMR spectrum of the imidazolidinone catalyst $\mathbf{1}$ is shown in Figure 2. Students found the assignment of catalyst ${ }^{1} \mathrm{H}$ NMR signals relatively straightforward.

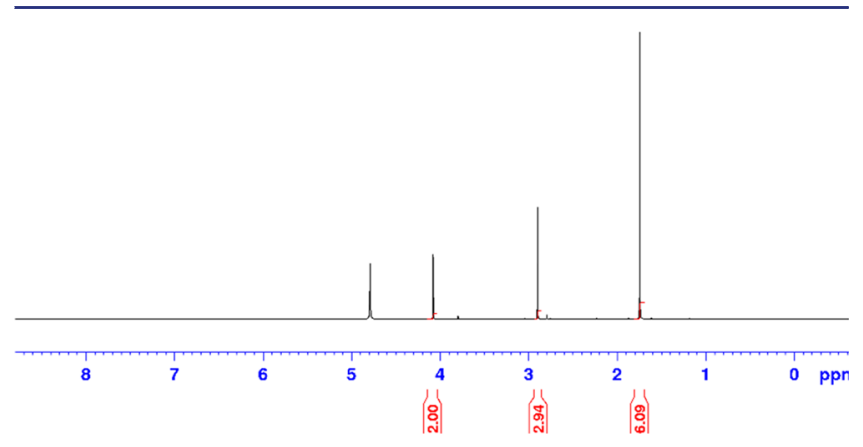

Figure 2. A student's ${ }^{1} \mathrm{H}$ NMR spectrum of imidazolidinone catalyst 1 $\left(300 \mathrm{MHz}, \mathrm{D}_{2} \mathrm{O}\right)$.

The second part of the experiment allowed the students to utilize the organocatalyst in a Diels-Alder cycloaddition. The catalyst operates via iminium ion activation of the dienophile, an $\alpha, \beta$-unsaturated aldehyde. This iminium ion activation lowers the LUMO of the dienophile, promoting the $[4+2]$ cycloaddition. Catalyst 1 generated an endo/exo mixture of adduct 7 in high yields and did not require column chromatography. An example of a student's ${ }^{1} \mathrm{H}$ NMR spectrum of adduct 7 is shown in Figure 3. Students were able to assign key product ${ }^{1} \mathrm{H}$ NMR signals; e.g., aldehyde, olefin, aryl, and alkyl ${ }^{1} \mathrm{H}$ NMR signals.

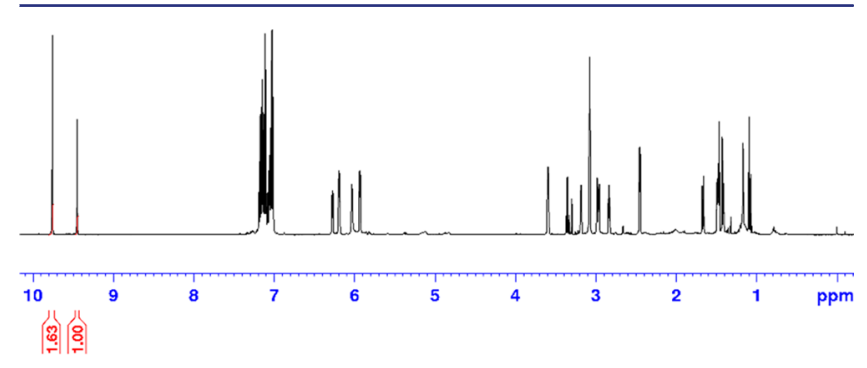

Figure 3. A student's ${ }^{1} \mathrm{H}$ NMR spectrum of adduct $7(300 \mathrm{MHz}$, $\left.\mathrm{CDCl}_{3}\right)$.

Both the endo and exo products were formed as a racemic mixture. The dr was measured using ${ }^{1} \mathrm{H}$ NMR spectroscopy by comparing the integration of the aldehyde peaks of both the endo and exo products (endo/exo ratio of $1 / 1.63$, Figure 3 ). The er was measured using chiral GC (Figure 4, see the Supporting Information for separation conditions). An experiment employing catalyst 2 was performed in advance by a teaching assistant and allowed a comparison to be made between the commercially available chiral imidazolidinone 2 (see Supporting Information) and the student-synthesized achiral imidazolidione 1. The commercial catalyst 2 generated the adduct 7 in high yields, with a dr of $1 / 1.42$ and an er of 96/4 for exo (major product) and 98.5/1.5 for endo (minor product) (Figure 4).

Comparing both Diels-Alder cycloadditions highlights the importance of organocatalyst structure in the stereochemical outcome of the reaction. Students can be directed to the literature to inform their write up discussion on the mechanism and selectivity. ${ }^{20,21}$ Additional explanation is given in the Supporting Information. 


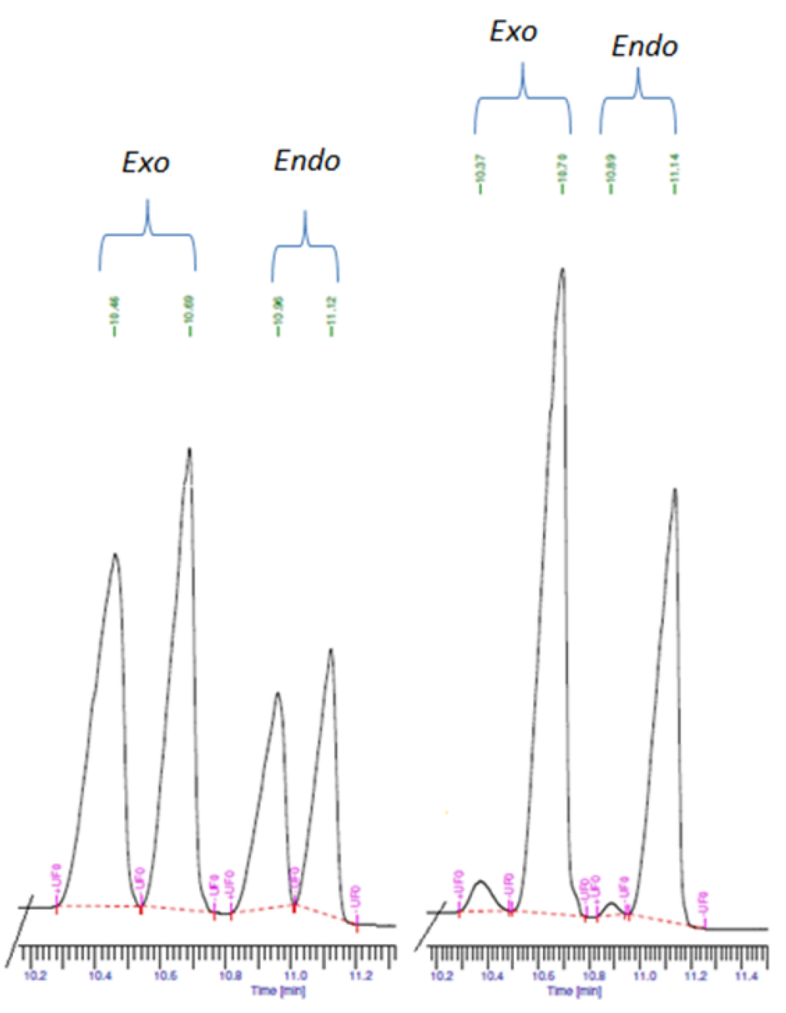

Figure 4. (a) GC trace of adduct 7 using catalyst 1. (b) GC trace of adduct 7 using catalyst 2 .

In conclusion, this laboratory practical takes place over three laboratory sessions and as such is ideal as a third- and fourthyear undergraduate lab. The experiments can be used to demonstrate a number of important topics in organic chemistry and several analytical techniques. As discussed, key topics covered include amide bond formation, aminal formation, iminium ion catalysis, and cycloaddition chemistry as well as NMR spectroscopy, GC, and TLC. State of the art methodologies for asymmetric induction can be reinforced, and links can be made to the concept of avoiding environmentally unfriendly metals and biochemistry (organocatalysts mimic some enzymatic reactions). As such, the practical can engage a variety of students. The mechanism explaining the stereochemical control is nontrivial, but a practical experience such as this will help students in their understanding of what can be challenging lecture material. The range of concepts and techniques employed allows the instructor to tailor the laboratory experience to best suit his/her course content.

\section{ASSOCIATED CONTENT}

\section{(S) Supporting Information}

The Supporting Information is available on the ACS Publications website at DOI: 10.1021/acs.jchemed.5b00812.

Instructions for students, safety information, sample spectra (GC and ${ }^{1} \mathrm{H}$ and ${ }^{13} \mathrm{C}$ NMR spectroscopy), and notes for instructors (PDF)

\section{AUTHOR INFORMATION}

\section{Corresponding Author}

*E-mail: john.stephens@nuim.ie.

\section{Notes}

The authors declare no competing financial interest.

\section{ACKNOWLEDGMENTS}

We thank the Irish Research Council for funding. We thank the Maynooth University Department of Chemistry for supporting this work. We also thank Walter Walsh at Maynooth University Department of Chemistry.

\section{REFERENCES}

(1) Ahrendt, K. A.; Borths, C. J.; MacMillan, D. W. C. New Strategies for Organic Catalysis: The First Highly Enantioselective Organocatalytic Diels-Alder Reaction. J. Am. Chem. Soc. 2000, 122 (17), 4243-4244.

(2) (a) Dalko, P. I. Enantioselective Organocatalysis: Reactions and Experimental Procedures; Wiley-VCH: Weinheim, Germany, 2007. (b) Dalko, P. I. Comprehensive Enantioselective Organocatalysis; WileyVCH: Weinheim, Germany, 2013.

(3) (a) Murphy, J. J.; Quintard, A.; McArdle, P.; Alexakis, A.; Stephens, J. C. Asymmetric Organocatalytic 1,6-Conjugate Addition of Aldehydes to Dienic Sulfones. Angew. Chem., Int. Ed. 2011, 50 (22), 5095-5098. (b) Pezzati, B.; Chellat, M. F.; Murphy, J. J.; Besnard, C.; Reginato, G.; Stephens, J. C.; Alexakis, A. Organocatalytic Asymmetric Annulation of 1,3-Bis(alkoxycarbonyl)buta-1,3-dienes and Aldehydes. Org. Lett. 2013, 15 (12), 2950-2953. (c) Halskov, K. S.; Donslund, B. S.; Barfüsser, S.; Jørgensen, K. A. Organocatalytic Asymmetric Formation of Steroids. Angew. Chem., Int. Ed. 2014, 53 (16), 41374141.

(4) Wade, E. O.; Walsh, K. E. A Multistep Organocatalysis Experiment for the Undergraduate Organic Laboratory: An Enantioselective Aldol Reaction Catalyzed by Methyl Prolinamide. J. Chem. Educ. 2011, 88 (8), 1152-1154.

(5) Bennett, G. D. A Green Enantioselective Aldol Condensation for the Undergraduate Organic Laboratory. J. Chem. Educ. 2006, 83 (12), $1871-1872$.

(6) Lazarski, K. E.; Rich, A. A.; Mascarenhas, C. M. A One-pot, Asymmetric Robinson Annulation in the Organic Chemistry Majors Laboratory. J. Chem. Educ. 2008, 85 (11), 1531-1534.

(7) Wong, T. C.; Sultana, C. M.; Vosburg, D. A. A Green, Enantioselective Synthesis of Warfarin for the Undergraduate Organic Laboratory. J. Chem. Educ. 2010, 87 (2), 194-195.

(8) Carroll, A.; Kavanagh, D. J.; McGovern, F. P.; Reilly, J. W.; Walsh, J. J. Nature's Chiral Catalyst and Anti-Malarial Agent: Isolation and Structure Elucidation of Cinchonine and Quinine from Cinchona calisaya. J. Chem. Educ. 2012, 89 (12), 1578-1581.

(9) Stacey, J.M.; Dicks, A. P.; Goodwin, A. A.; Rush, B. M.; Nigam, M. Green Carbonyl Condensation Reactions Demonstrating Solvent and Organocatalyst Recyclability. J. Chem. Educ. 2013, 90 (8), 10671070.

(10) Morgan, J. P.; Shrimp, J. H. N-Heterocyclic Carbene-Catalyzed Alcohol Acetylation: An Organic Experiment Using Organocatalysis. J. Chem. Educ. 2014, 91 (6), 911-914.

(11) Chan, J. M. W.; Zhang, X.; Brennan, M. K.; Sardon, H.; Engler, A. C.; Fox, C. H.; Frank, C. W.; Waymouth, R. M.; Hedrick, J. L. Organocatalytic Ring-Opening Polymerization of Trimethylene Carbonate To Yield a Biodegradable Polycarbonate. J. Chem. Educ. 2015, 92 (4), 708-713.

(12) Snider, B. B. N-Heterocyclic Carbene-Catalyzed Reaction of Chalcone and Cinnamaldehyde To Give 1,3,4-Triphenylcyclopentene Using Organocatalysis To Form a Homoenolate Equivalent. J. Chem. Educ. 2015, 92 (8), 1394-1397.

(13) Monge, D. Alkaloid-Derived Thioureas in Asymmetric Organocatalysis: A Cooperative Learning Activity in a Project-Based Laboratory Course. J. Chem. Educ. 2015, 92 (8), 1390-1393.

(14) Walsh, K. E.; Wade, E. O. Organocatalytic Enantioselective 1,4Addition as a Capstone Experiment in Organic Chemistry. Chem. Educ. 2012, 17, 223-225.

(15) Lelais, G.; MacMillan, D. W. C. Modern Strategies in Organic Catalysis: The Advent and Development of Iminium Activation. Aldrichimica Acta 2006, 39 (3), 79-87. 
(16) Sieck, S. R. Tamiflu: An Advanced Organic Chemistry Laboratory in Multi-Step Synthesis. Chem. Educ. 2013, 18, 110-115. (17) Samulis, L.; Tomkinson, N. C. O. Preparation of the MacMillan Imidazolidinones. Tetrahedron 2011, 67 (23), 4263-4267.

(18) Dickson, R. S.; Dobney, B. J.; Eastwood, F. W. Preparation of Cyclopentadiene from its Dimer. J. Chem. Educ. 1987, 64 (10), 898.

(19) Groselj, U.; Schweizer, W. B.; Ebert, M.-O.; Seebach, D. 5Benzyl-3-methylimidazolidin-4-one Derived Reactive Intermediates of Organocatalysis - A Comforting Resemblance of X-ray, NMR, and DFT Solid-phase, Liquid-phase, and Gas-phase Structures. Helv. Chim. Acta 2009, 92 (1), 1-13.

(20) Brazier, J. B.; Evans, G.; Gibbs, T. J. K.; Coles, S. J.; Hursthouse, M. B.; Platts, J. A.; Tomkinson, N. C. O. Solution Phase, Solid State, and Theoretical Investigations on the MacMillan Imidazolidinone. Org. Lett. 2009, 11 (1), 133-136.

(21) Erkkilä, A.; Majander, I.; Pihko, P. M. Iminium Catalysis. Chem. Rev. 2007, 107 (12), 5416-5470. 